\title{
p-Coumaric acid has an ameliorative effect on peptic ulcer: a macroscopic, microscopic and biochemical analysis
}

\author{
Meltem KOLGAZI ${ }^{1}$ (D), Gulsen OZTOSUN ${ }^{(\mathbb{D})}$, Muge YALCIN ${ }^{2}$ (D), Zehra Neslisah UNAN² (D), Edanur ARSOY2 (D), Simge ORAL ${ }^{3}$ (D), \\ Sumeyye CILINGIR ${ }^{1}$ (D), Merve ACIKEL ELMAS ${ }^{4}$ D, Serap ARBAK ${ }^{4}$ (D) \\ ${ }^{1}$ Department of Physiology, School of Medicine, Acibadem Mehmet Ali Aydinlar University, Istanbul, Turkey. \\ ${ }^{2}$ Medical Student, School of Medicine, Acibadem Mehmet Ali Aydinlar University, Istanbul, Turkey. \\ ${ }^{3}$ Medical Student, School of Medicine, Yeditepe University, Istanbul, Turkey. \\ ${ }^{4}$ Department of Histology and Embryology, School of Medicine, Acibadem Mehmet Ali Aydinlar University, Istanbul, Turkey.
}

Corresponding Author: Meltem KOLGAZI
E-mail: meltem.kolgazi@acibadem.edu.tr

Submitted: 17.08.2020 Accepted: 02.11.2020

\section{ABSTRACT}

Objective: p-Coumaric acid is commonly found in edible plants in nature and is known to be an effective antioxidant. This study aimed to investigate the therapeutic effects of p-coumaric acid on ethanol-induced gastric ulcer model

Materials and Methods: After an 18-hour starvation period, the ulcer was induced in male Sprague-Dawley (250-300) rats by intragastric administration of $75 \%$ ethanol. An hour after ulcer induction, $\mathrm{p}$-coumaric acid ( $250 \mathrm{mg} / \mathrm{kg})$ suspended in $1 \mathrm{ml}$ tween-80 was administered intragastrically. The control and ulcer groups received $1 \mathrm{ml}$ tween-80. One hour later, all rats were euthanized and stomach samples were collected for macroscopic examination, histological evaluation, and measurement of myeloperoxidase (MPO) activity, malondialdehyde (MDA), and glutathione (GSH) levels.

Results: Ethanol induction resulted in gastric epithelial and mucosal injury, increased MPO activity and MDA levels; and decreased GSH levels. Treatment with p-coumaric acid at a dose of $250 \mathrm{mg} / \mathrm{kg}$ markedly improved the gastric injury macroscopically, microscopically, and biochemically due to decreasing MPO activity and MDA levels while increasing GSH levels.

Conclusion: p-Coumaric acid markedly ameliorated the gastric epithelial and mucosal injury induced by ethanol. The therapeutic effects of p-coumaric acid could be due to its antioxidant properties and protective role against GSH depletion and neutrophil accumulation.

Keywords: p-Coumaric acid, Ulcer, Ethanol, Antioxidant, Neutrophil

\section{INTRODUCTION}

p-Coumaric acid is one of the three isomers of coumaric acid, which is a member of the hydroxycinnamic acid family. It is found in a variety of edible plants, vegetables, vinegar, wine, and barley grain in free or bound form. p-Coumaric acid and other phenolic acids are known to be powerful antioxidants [1]. Recent interest in phenolic acids stems from their potential capacity to diminish tissue damage caused by oxidative stress [2]. It has been discovered that pre-treatment of animals with p-coumaric acid is helpful in reducing cerebral ischemia reperfusion (IR) induced injuries, oxidative stress, size of infarction and neuronal vulnerability to death [3]. Moreover, it has been proposed that $\mathrm{p}$-coumaric acid can block oxidative modification of low-density lipoprotein (LDL), which is a necessary step in atherosclerosis [4]. p-Coumaric acid also suppresses dyslipidemia, hepatosteatosis and oxidative stress in obese rats and has an adipogenic effect [5]. Recent investigations have also shown that p-coumaric acid has anti-cancer [6,7] and anti-inflammatory [8] effects. Recently, reduction in acid proinflammatory cytokines [tumor necrosis factor- $\alpha$, interleukin (IL)-1 $\beta$, IL-6, and IL-17], and inflammatory enzymes (inducible nitric oxide and cyclooxygenase-2) by p-coumaric acid has been shown in arthritic rats [9]. Kilani-Jaziri S, et al., demonstrated that p-coumaric acid augments the killing activity of isolated natural killer cells and cytotoxic T cells, and possibly activates B cells along with enhanced humoral immunity [10].

Ulcer is defined as a disruption in the mucosa, which extends through the muscularis mucosae, and is surrounded by acute and chronic inflammation. Each year, peptic ulcer disease affects 4 million people around the world [11]. Although, the pathophysiology is not yet fully understood, it is accepted that peptic ulcer results from an imbalance between defensive mechanisms of the mucosa and other aggressive factors

How to cite this article: Kolgazi M, Oztosun G, Yalcin M, et al. p-Coumaric acid has an ameliorative effect on peptic ulcer: a macroscopic, microscopic and biochemical analysis. Marmara Med J 2021; 34(1):6-11. doi: 10.5472/marumj.859006 
[12]. Acid secretion, pepsin secretion, and parietal cells are among aggravating factors; whereas mucus secretion, mucosal regeneration, prostaglandins and gastric blood flow are examples of protective factors [13]. Some factors such as excessive alcohol consumption, $\mathrm{H}$. pylori infection and prolonged nonsteroid anti-inflammatory drug treatment destroy the balance, which is easier in genetically predisposed individuals.Ingestion of excessive ethanol could induce gastric mucosal injury by stimulating mucosal epithelial cell apoptosis, inflammatory reaction and oxidative stress in gastric tissue [14]. Ethanol solubilizes the protective mucus via proteolytic and hydrolytic actions, and increases the concentration of hydrochloric acid and pepsin. It produces reactive oxygen species (ROS) that alter cell structure and function. These factors eventually damage the membrane and lead to ulceration [15]. Anti-oxidant agents are effective in peptic ulcer disease treatment due to their ROS scavenging and cytoprotective activities. No previous study has analyzed the anti-ulcer effects of p-coumaric acid at microscopic and biochemical levels, which makes this study unique in terms of revealing the underlying mechanisms.

Our aim was to determine the therapeutic effects of p-coumaric acid on ethanol-induced gastric ulcer models at macroscopic, microscopic and biochemical levels.

\section{MATERIAL and METHODS}

\section{Animals}

Sprague-Dawley rats were provided by the Acibadem Mehmet Ali Aydinlar University (ACU) Animal Center. Male rats (300$350 \mathrm{~g} ; \mathrm{n}=7-8$ per group) were housed at a constant temperature $\left(22 \pm 1^{\circ} \mathrm{C}\right)$ at a humidity of $65-70 \%$ with $12 \mathrm{~h}$ light/dark cycles. Rats were fed standard pellet chow and water ad libitum. All protocols were approved by ACU Local Ethics Committee for Animal Experiments (ACU-HADYEK 2017/2).

\section{Induction of ulcer and treatment protocol}

Following 18-h starvation, rats were divided into 4 groups. Rats were given $75 \%$ ethanol (ulcer and ulcer + p-coumaric acid groups) or saline (control and p-coumaric acid groups) by oral gavage under light isoflurane anesthesia. One hour before ethanol or saline administration, rats were treated with either $1 \mathrm{ml}$ 10\% tween-80 (ulcer and control groups) as vehicle or p-coumaric acid (Sigma-Aldrich C9008, Merck, Germany; $250 \mathrm{mg} / \mathrm{kg}$; suspended in 10\% tween-80) (p-coumaric acid and ulcer + p-coumaric acid groups) per oral (Table I). Barros, et al., showed that the dose of $250 \mathrm{mg} / \mathrm{kg}$ of $\mathrm{p}$-coumaric acid reduced the macroscopic lesion index most compared to other doses [16]. Therefore, the dose of $250 \mathrm{mg} / \mathrm{kg}$ p-coumaric acid was used for treatment in the study. One hour after ulcer induction, all rats were euthanized by cutting the aorta and removing the heart under deep isoflurane anesthesia. Stomach samples were collected for macroscopic examination, histological evaluation, microscopic scoring and biochemical analyses. For histological evaluation the stomach samples were examined under light microscopy and for biochemical analyses tissue-associated myeloperoxidase (MPO) activity as the indicator of neutrophil infiltration, malondialdehyde (MDA) which is the end-product of lipid peroxidation and endogen antioxidant glutathione (GSH) levels were measured.

Table I. Experimental protocol
\begin{tabular}{|l|l|l|l|}
\hline & $\mathbf{1 0 : 0 0}$ & $\mathbf{1 1 : 0 0}$ & $\mathbf{1 2 : 0 0}$ \\
\hline Control & Tween-80 & Saline & Euthanasia \\
\hline p-coumaric acid & p-coumaric acid $250 \mathrm{mg} / \mathrm{kg}$ & Saline & Euthanasia \\
\hline Ulcer & Tween- 80 & Ethanol & Euthanasia \\
\hline $\begin{array}{l}\text { Ulcer + p-coumaric } \\
\text { acid }\end{array}$ & p-coumaric acid $250 \mathrm{mg} / \mathrm{kg}$ & Ethanol & Euthanasia \\
\hline
\end{tabular}

\section{Macroscopic scoring}

Macroscopic scoring was performed by an observer who was blind to the study using a semi-quantitative scale of 0 to 3 . A score of 0 indicated a normal mucosa. A score of 1 indicated that there were a total number of 1 to 4 small petechiae observed on the stomach. A score of 2 indicated 5 or more petechiae or hemorrhagic streaks up to 4 millimeters in length. A score of 3 indicated erosions longer than 5 millimeters, or confluent hemorrhages [17].

\section{Histological evaluation and microscopic scoring}

For light microscopic examination, stomach samples were placed in $10 \%$ formalin solution, dehydrated through ascending alcohol series (70\%, 90\%, 96\% and 100\%), cleared in xylene and embedded in paraffin. For each animal, four randomly taken tissue sections $(5 \mu \mathrm{m})$ were stained with hematoxylin and eosin $(\mathrm{H} \& \mathrm{E})$. The slides were examined under a AxioCam MRc5 photomicroscope (Zeiss, Germany). The photos of the samples were taken via the camera of the photomicroscope itself. The criteria for scoring were epithelial desquamation, mucosal hemorrhage, glandular damage and eosinophilic infiltration. Each specimen was scored using a scale ranging from 0 to 3 (0: none, 1: mild, 2: moderate, and 3: severe) for each criterion. The total score was 12. Histologic examination and scoring were performed by an observer (S.A.) who was unaware of the treatment groups [18].

\section{Measurement of MDA and GSH levels}

Stomach samples were homogenized in 10 volumes of ice-cold $10 \%$ trichloroacetic acid and centrifuged at $3000 \mathrm{rpm}$ for 15 $\min$ at $4{ }^{\circ} \mathrm{C}$ and then removed supernatants were re-centrifuged at $9000 \mathrm{rpm}$ at $4{ }^{\circ} \mathrm{C}$ for $8 \mathrm{~min}$. In order to detect MDA levels, the samples combined with thiobarbituric acid boiled for 15 minutes. Lipid peroxide levels were expressed in terms of MDA equivalents as nmol MDA per $g$ tissue [19]. GSH was determined by a spectrophotometric method which is the modification of Ellman procedure [20]. Briefly, removed supernatant was added to a mixture composed of $2 \mathrm{ml}$ of $0.3 \mathrm{~mol} / \mathrm{l} \mathrm{Na}_{2} \mathrm{HPO}_{4}-2 \mathrm{H}_{2} \mathrm{O}$ solution and $0.2 \mathrm{ml}$ dithiobis-nitrobenzoate solution $(0.4 \mathrm{mg} /$ $\mathrm{ml}$ in $1 \%$ sodium citrate). Immediately, the absorbance of the mixture was measured at $412 \mathrm{~nm}$. GSH levels were calculated using an extinction coefficient of $1.36 \times 10^{4} \mathrm{M} / \mathrm{cm}$. Results are expressed in micromoles GSH per gram tissue. 


\section{Measurement of MPO activity}

Myeloperoxidase activity, an indicator of neutrophil accumulation, was assessed by measuring the $\mathrm{H}_{2} \mathrm{O}_{2}$-dependent oxidation of o-dianisidine $2 \mathrm{HCl}$. One unit of enzyme activity was defined as the amount of MPO present that causes a change in absorbance of 1.0 unit $\mathrm{min}^{-1}$ at $460 \mathrm{~nm}$ and $37^{\circ} \mathrm{C}$ and expressed in units per g tissue [21]. Gastric tissue samples were homogenized for a minute in a solution containing potassium phosphate buffer $\left(\mathrm{K}_{2} \mathrm{HPO}_{4}\right)$ and hexadecyltrimethylammonium bromide (HETAB). They were centrifuged at $12000 \mathrm{rpm}$ at $4{ }^{\circ} \mathrm{C}$ for 10 minutes and the supernatants were discarded. Then, pellets were mixed with a solution of $\mathrm{K}_{2} \mathrm{HPO}_{4}$, HETAB and Ethylenediaminetetraacetic acid (EDTA) with rehomogenization for 15 seconds. Finally, oxidation reaction of o-Dianisidine in the presence of $\mathrm{H}_{2} \mathrm{O}_{2}$ was conducted with the mixture and MPO activity was measured spectrophotometrically according to the reaction. MPO activity was expressed as U/g tissue.

\section{Statistical Analysis}

All data expressed as means \pm S.E. parameters were compared using two-way analysis of variance (ANOVA) followed by Tukey-Kramer multiple comparison tests. Values of $\mathrm{p}<0.05$ were regarded as significant. Calculations were done using the InStat statistical analysis package (GraphPad Software, San Diego, CA, USA).

\section{RESULTS}

\section{Macroscopic and microscopic lesion scores}

When compared with the vehicle-treated control group, the ethanol-induced ulcer group had significantly high macroscopic $(\mathrm{p}<0.001)$ and microscopic $(\mathrm{p}<0.001)$ lesion scores (Fig.1). When compared with the untreated ethanol-induced ulcer group, the ulcer group treated with p-coumaric acid had significantly reduced macroscopic $(2.55 \pm 0.20$ and $1.4 \pm 0.4$; respectively) $(\mathrm{p}<0.05)$ and microscopic $(10.33 \pm 0.62$ and $3.17 \pm 0.6$; respectively) $(\mathrm{p}<0.001)$ lesion scores (Fig.1). In terms of macroscopic scoring, the ulcer group had severe hemorrhage and a lot of petechiae. Treatment with p-coumaric acid decreased the gastric lesions observed macroscopically and reduced the macroscopic lesion score significantly when compared with the untreated ulcer group. The ulcer-free group treated only with p-coumaric acid $(0.17 \pm 0.02)$ had a similar macroscopic lesion score with the control (Fig.1A). In microscopic examination p-coumaric acid treatment also decreased the microscopic scores compared with ulcer group given vehicle. Unexpectedly, only p-coumaric acid given rats $(4.33 \pm 0.33)$ had slightly higher microscopic score than control group (Fig.1B). This might be driven from slight inflammatory cell infiltration due to the effect of p-coumaric acid that increases blood flow.

In terms of histological evaluation, the control group had regular gastric mucosa with surface epithelium and glandular epithelium. In the ulcer group, there was severe damage of surface epithelium and glandular epithelium with hemorrhagic regions and desquamated cells. The ulcer-free group treated with only p-coumaric acid had slight degeneration of gastric epithelium and moderate inflammatory cell infiltration. The ulcer group treated with p-coumaric acid showed minor damage at surface epithelium and gastric glands, and eosinophilic infiltration was reduced significantly (Fig. 2).

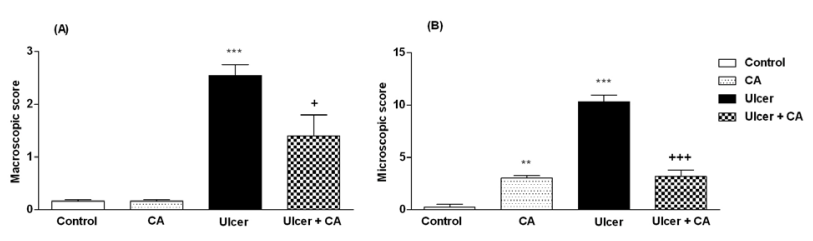

Fig 1. Macroscopic (A) and microscopic (B) lesion scores in control, coumaric acid (CA), ulcer and ulcer + coumaric acid (ulcer $+C A)$ groups. ${ }^{* * *} p<0.001$ compared with control group. $+p<0.05,+++p<0.001$ compared with ulcer group. The results are expressed as the mean \pm S.E.M.
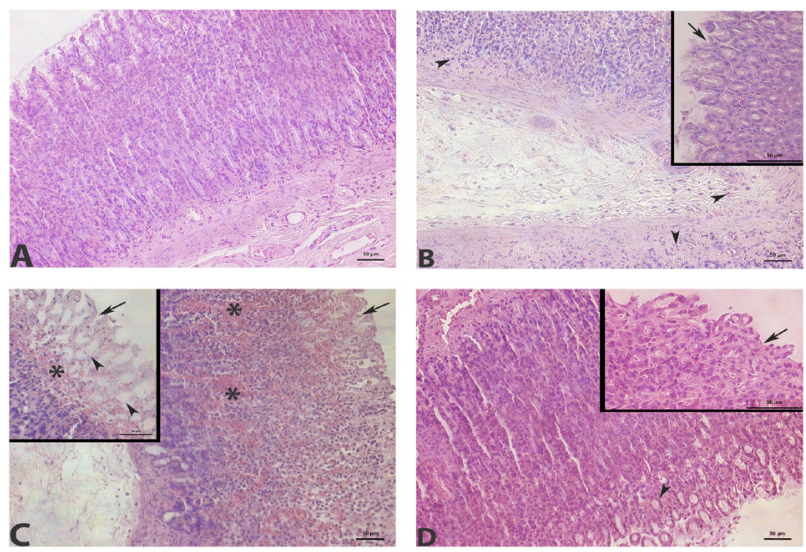

Fig 2. Histological analysis of the tissue samples by hematoxylin and eosin staining (A-D). (A) Regular gastric mucosa with surface epithelium and glandular epithelium in the control group. (B) Slight degeneration of gastric epithelium $(\rightarrow)$ and moderate inflammatory cell infiltration $(\rightarrow)$ in the coumaric acid group. (C) Severe damage of surface epithelium $(\rightarrow)$ and glandular epithelium ( ) with hemorrhagic regions $\left(^{*}\right)$ and desquamated cells in the ulcer group. (D) Minor damage at surface epithelium $(\rightarrow)$ and gastric glands $(\longrightarrow)$ in the ulcer combined with coumaric acid treatment group. HeE staining, scale bars: $50 \mu \mathrm{m}$ (x20), inset: $20 \mu \mathrm{m}$ (x40).

\section{MDA and GSH levels}

As expected, the ulcer group was characterized by a significant increase in gastric MDA level along with a concomitant decrease in GSH content. Increase in MDA (13.07 \pm 0.88$)$ with ethanol administration considering the control group $(6.99 \pm 0.97)(\mathrm{p}<0.001)$ decreased significantly with $\mathrm{p}$-coumaric acid treatment $(8.85 \pm 0.43)(\mathrm{p}<0.01)($ Fig. $3 \mathrm{~A})$. The endogenous antioxidant GSH was lower in the ulcer group (1.06 \pm 0.06$)$ compared to the control $(1.89 \pm 0.09)(\mathrm{p}<0.05)$ and treatment with 
p-coumaric acid $(2.01 \pm 0.28)$ prevented gastric GSH depletion $(\mathrm{p}<0.05)$ (Fig. $3 \mathrm{~B})$. Only $\mathrm{p}$-coumaric acid administration had similar values with control group for both MDA and GSH measurement (9.32 \pm 0.73 and $1.68 \pm 0.18$; respectively).
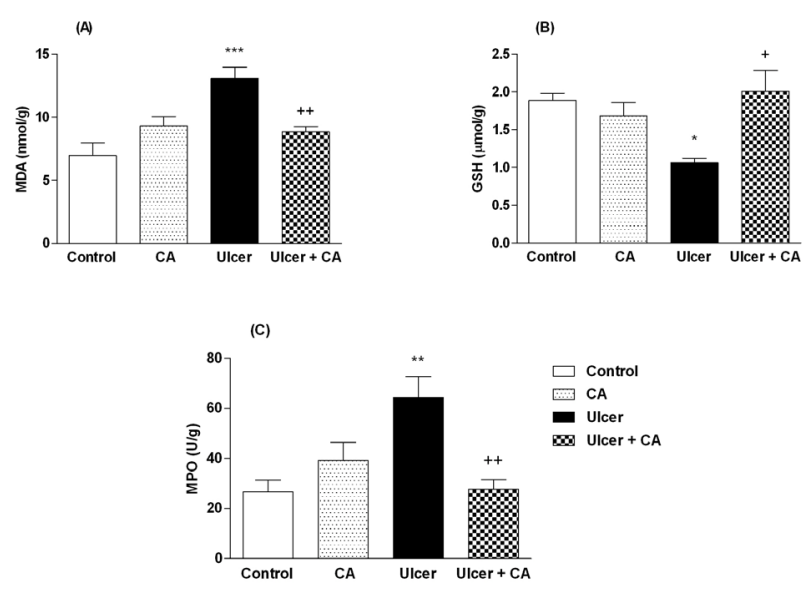

Fig 3. (A) Malondialdehyde (MDA) levels, (B) Glutathione (GSH) levels, (C) Myeloperoxidase (MPO) activity in control, coumaric acid (CA), ulcer and ulcer + coumaric acid (ulcer $+C A$ ) groups determined by procedures explained above. ${ }^{*} p<0.05,{ }^{* *} p<0.01,{ }^{* *} p<0.001$ compared to the control group. $+p<0.05,++p<0.01$ compared to the ulcer group. The results are expressed as the mean \pm S.E.M.

\section{MPO activity}

Ethanol administration resulted in an increase in MPO activity in the ulcer group $(64.22 \pm 8.57)$ compared with control group $(26.69 \pm 4.55) \quad(\mathrm{p}<0.01)$. Elevated MPO activity was reduced by the p-coumaric acid treatment $(27.7 \pm 3.8)$ significantly $(\mathrm{p}<0.01)$ (Fig.3C). p-Coumaric acid treatment without ethanol administration (39.16 \pm 7.2$)$ increased MPO activity slightly; however there was no significant difference with control group.

\section{DISCUSSION}

Peptic ulcer is a disease characterized by an imbalance between the factors that damages the mucosa and those for its protection, resulting in a lesion of the lining of the upper digestive tract. When the gastric mucosa is exposed to noxious agents such as alcohol, the extent of gastric damage depends upon the balance between the factors [22]. p-Coumaric acid is widely distributed in plants and mushrooms, and shows various biological activities as anti-oxidant, anti-tumorogenesis and anti-inflammatory effects [23].The results of the study demonstrate that p-coumaric acid treatment markedly improved the ethanol induced gastric injury, as confirmed by histological evaluation and biochemical assays as a measure of the extent of the inflammatory response. The extent of injury was significantly reduced by $\mathrm{p}$-coumaric acid treatment as assessed by macroscopic and microscopic scores. MPO activity as a marker of recruitment of neutrophils was suppressed in p-coumaric acid treated group. Moreover, p-coumaric acid treatment reduced MDA content increased by ethanol administration and gastric GSH levels, which were depleted in ethanol consumed rats, were preserved with p-coumaric acid treatment.

p-Coumaric acid is a hydroxycinnamic acid derivative that was found to be an effective antioxidant in different in vitro assays including superoxide anion radical scavenging, hydrogen peroxide scavenging and metal chelating activities [24]. As shown before, $250 \mathrm{mg} / \mathrm{kg}$ p-coumaric acid diminished the lesion index, the total area of the lesion and the percentage of lesion in ulcer induced rats. However, anti-ulcer activity of p-coumaric acid at the microscopic level and the biochemical parameters related to inflammation were investigated for the first time in the present study. The current study revealed that p-coumaric acid decreased macroscopic and microscopic scores and histological results demonstrated that the ingestion of p-coumaric acid ameliorated the gastric injury induced by ethanol.

Ethanol-induced gastric injury is a key experimental model commonly utilized for preclinical assessment of agents with potential anti-ulcer activity since ethanol has been regarded as a leading cause of gastric ulcer in humans [25]. The effect of ethanol on gastric mucosa is a complicated process and it is associated with multiple pathologies. Ethanol has the tendency to create ulcers via oxidative pathways, which lead to solubilization of protective mucus, increase in hydrochloric acid $(\mathrm{HCl})$ and pepsin concentrations and cell apoptosis in gastric tissue. The result of one of these oxidative pathways is the production of ROS, which play a major role in the ulcer mechanism. ROS decrease the $\mathrm{pH}$ levels in stomach and speed up the oxidation process, therefore causing ulceration in the mucosa [26].

There are several potential sources of ROS production by the inflamed tissues. These may include the epithelial cells, the microvascular endothelium or the inflammatory cells. As the inflammatory cells, activated neutrophils can induce tissue injury via the release of various toxic metabolites, including ROS and proteases [27]. Neutrophil infiltration to injured gastric mucosa can be indicated by MPO activity. MPO, found in lysosomes of neutrophils, monocytes and macrophages, is one of the enzymes responsible for catalyzing ROS generation [28]. As shown before, these harmful actions can be blocked by antioxidant substances which scavenge the free radicals and detoxify the organism [29]. In the present study, increased MPO levels with ethanol administration were depressed with the p-coumaric acid treatment. This decline in the MPO activity which represents the protective effects of p-coumaric acid against neutrophil accumulation in gastric tissue was shown for the first time in this study in the literature.

Meanwhile, ROS generated by activated leukocytes trigger mucosal damage via lipid peroxidation and via depletion of the antioxidant defenses such as reduced GSH. Highly reactive compound, MDA, produced by peroxidation of lipids also increases during formation of ethanol induced ulcers [30]. In the study increased MDA levels because of ethanol ingestion were reduced by p-coumaric acid treatment. On behalf of its antioxidant effects, p-coumaric acid decreased MDA levels, possibly via its scavenging effect [31]. 
Glutathione levels were preserved in the p-coumaric acid treated ulcer group. This suggests that the protective effects of $\mathrm{p}$-coumaric acid include prevention of the depletion of GSH, which is an endogen antioxidant. GSH content in gastric tissue reveals anti-oxidant capacity and it is used as marker for tissue injury and oxidative stress. In the present study depleted gastric GSH with the ethanol administration was preserved by p-coumaric acid. Due to its reducing properties, GSH protects the cells against injuries promoted by free radicals, radiation, ultraviolet light, besides removing products of lipid peroxidation [32].

As a result, our study showed the beneficial and anti-ulcer effect of p-coumaric acid against ethanol induced gastric damage. The healing effects of p-coumaric acid could be due to its preventive effect of neutrophil accumulation, lipid peroxidation and GSH depletion. On the basis of our findings, it could be suggested that p-coumaric acid can be a therapeutic choice for gastric ulcer.

\section{Acknowledgements}

The authors are grateful to Acibadem Mehmet Ali Aydinlar University Laboratory Animal Application and Research Center (ACU-DEHAM) for supplying the rats used in the study.

\section{Compliance with Ethical Standards}

Ethical Approval: All protocols were approved by ACU Local Ethics Committee for Animal Experiments (ACU-HADYEK 2017/2).

Financial Support: The authors have no relevant financial information to disclose.

Conflict of Interest: The authors declare that there are no conflicts of interest.

Author Contributions: M.K. and G.O. contributed to the conception and design. M.K., G.O., M.Y., Z.N.U., E.A., S.O., S.C., M.A.E, N.A. performed experiments and did data collection, M.K. and S.A. analyzed data. M.K., G.O., M.Y., Z.N.U., E.A., S.O., S.A. contributed to the writing and M.K. did the critical revision of the article. All authors approved the final version of the article.

\section{REFERENCES}

[1] Pei K, Ou J, Huang J, Ou S. p-Coumaric acid and its conjugates: dietary sources, pharmacokinetic properties and biological activities. J Sci Food Agric 2015; 96:2952-62. doi: 10.1002/ jsfa. 7578

[2] Goleniowski M, Bonfill M, Cusido RM, Palazon J. Phenolic Acids. Natural Products. 2013 Springer-Verlag Berlin Heidelberg:1951-1973. doi: 10.1007/978-3-642-22144-6_64

[3] Sakamula R, Thong-Asa W. Neuroprotective effect of p-coumaric acid in mice with cerebral ischemia reperfusion injuries. Metab Brain Dis 2018; 33:765-73. doi: 10.1007/ s11011.018.0185-7

[4] Zang LY, Cosma G, Gardner H, Shi X, Castranova V, Vallyathan V. Effect of antioxidant protection by p-coumaric acid on low-density lipoprotein cholesterol oxidation. Am
J Physiol Cell Physiol 2000; 279:C954-60. doi: 10.1152/ ajpcell.2000.279.4.C954

[5] Ragab SM, AbdElghaffar SK, El-Metwally TH, Badr G, Mahmoud MH, Omar HM. Effect of a high fat, high sucrose diet on the promotion of non-alcoholic fatty liver disease in male rats: the ameliorative role of three natural compounds. Lipids Health Dis 2015; 14: 83. doi: 10.1186/s12944.015.0087-1

[6] Loo G. Redox-sensitive mechanisms of phytochemicalmediated inhibition of cancer cell proliferation. J Nutr Biochem 2003; 14:64-73. doi: 10.1016/S0955-2863(02)00251-6

[7] Sharma SH, Rajamanickam V, Nagarajan S. Antiproliferative effect of p-Coumaric acid targets UPR activation by downregulating Grp78 in colon cancer. ChemBiol Interact 2018; 291:16-28. doi: 10.1016/j.cbi/2018.06.001

[8] Pragasam SJ, Venkatesan V, Rasool M. Immunomodulatory and anti-inflammatory effect of p-coumaric acid, a common dietary polyphenol on experimental inflammation in rats. Inflammation 2013; 36:169-176. doi: 10.1007/ s10753.012.9532-8

[9] Neog MK, PragasamSJ, Krishnan M, Rasool M. p囚Coumaric acid, a dietary polyphenol ameliorates inflammation and curtails cartilage and bone erosion in the rheumatoid arthritis rat model. Biofactors 2017; 43:698-717. doi: 10.1002/biof.1377

[10] Kilani-Jaziri S, Mokdad-Bzeouich I, Krifa M, Nasr N, Ghedira $\mathrm{K}$, Chekir-Ghedira L. Immunomodulatory and cellular antioxidant activities of caffeic, ferulic, and p-coumaric phenolic acids: a structure-activity relationship study. Drug ChemToxicol 2017; 40:416-24. doi: 10.1080/01480.545.2016.1252919

[11] Thorsen K, Søreide JA, Kvaløy JT, Glomsaker T, Søreide K. Epidemiology of perforated peptic ulcer: Age - and gender-adjusted analysis of incidence and mortality. World J Gastroenterol 2013; 19:347-54. doi: 10.3748/wjg.v109.i3.347

[12] Salena BJ, Hunt RH. The stomach and duodenum. First Principles of Gastroenterology: the Basis of Disease and an Approach to Management. Mississauga, Canada: Canadian Association of Gastroenterology/Astra Pharmaceuticals, 1994:135-181.

[13] Adinortey MB, Ansah C, Galyuon I, Nyarko A. In Vivo models used for evaluation of potential antigastroduodenal ulcer agents. Ulcers 2013; 2013:1-12. doi: 10.1155/2013/796405

[14] Shen Y, Sun J, Niu C, et al. Mechanistic evaluation of gastroprotective effects of Kangfuxin on ethanol-induced gastric ulcer in mice. Chem Biol Interact 2017; 273:115-24. doi: 10.1016/j.cbi.2017.06.007

[15] Kwiecień S, Brzozowski T, Konturek SJ. Effects of reactive oxygen species action on gastric mucosa in various models of mucosal injury. J Physiol Pharmacol 2002; 53:39-50. PMID: 11939718

[16] Barros MP, Lemos M, Maistro EL, et al. Evaluation of antiulcer activity of the main phenolic acids found in Brazilian Green Propolis. J Ethnopharmacol 2008; 120:372-7. doi: 10.1016/j. jep.2008.09.015

[17] Szabo S, Trier JS, Brown A, Schnoor J, Homan HD, Bradford JC. A quantitative method for assessing the extent of experimental 
gastric erosions and ulcers. J Pharmacol Methods 1985; 13:5966. doi: 10.1016/0160-5402(85)90068-3

[18] Jahovic N, Erkanli G, Işeri S, Arbak S, Alican I. Gastric protection by alpha-melanocyte-stimulating hormone against ethanol in rats: involvement of somatostatin. Life Sci 2007; 80:1040-5. doi: 10.1016/j.lfs.2006.11.036

[19] Casini A, Ferrali M, Pompella AS, Maellaro E, Comporti M. Lipid peroxidation and cellular damage in extrahepatic tissues of bromobenzene intoxicated mice. Am. J. Pathol 1986; 123:520-31.

[20] Aykac G, Uysal M, Yalcin AS, Kocak-Toker N, Sivas A, $\mathrm{Oz} \mathrm{H}$. The effect of chronic ethanol ingestion on hepatic lipidperoxide, glutathione peroxidase and glutathione transferase in rat. Toxicology 1985; 46:71-6. doi: 10.1016/0300483X(85)90008-3

[21] Bradley PP, Preibat D, Christerser RD, Rothstein G. Measurement of cutaneous inflammation, estimation of neutrophil content with an enzyme marker. J. Invest. Dermatol 1982; 78:206-9. doi: 0022-202X/82/7803-0206\$02.00/0

[22] Vomero ND, Colpo E. Nutritional care in peptic ulcer. Arq Bras Cir Dig 2014; 27:298-302. doi: 10.1590/ S0102.672.0201400.040.0017

[23] Alam MA, Subhan N, Hossain H, et al. Hydroxycinnamic acid derivatives: a potential class of natural compounds for the management of lipid metabolism and obesity. Nutr Metab (Lond) 2016; 13:27. doi: 10.1186/s12986.016.0080-3

[24] Kiliç I, Yeşiloğlu Y. Spectroscopic studies on the antioxidant activity of p-coumaric acid. Spectrochim Acta A Mol Biomol Spectrosc 2013; 115:719-24. doi: 10.1016/j.saa.2013.06.110
[25] Liu Y, Tian X, Gou L, et al. Protective effect of 1-citrulline against ethanol-induced gastric ulcer in rats. Environ Toxicol Pharmacol 2012; 34:280-7. doi: 10.1016/j.etap.2012.04.009

[26] Brzozowski T, Konturek PC, Konturek SJ, et al. The role of melatonin and L-tryptophan in prevention of acute gastric lesions induced by stress, ethanol, ischemia, and aspirin. J. Pineal Res 1997; 23:79-89. doi: 10.1111/j.1600.079X.1997. tb00339.x

[27] Wright HL, Moots RJ, Bucknall RC, Edwards SW. Neutrophil function in inflammation and inflammatory diseases. Rheumatology (Oxford) 2010; 49:1618-31. doi: 10.1093/ rheumatology/keq045

[28] Bhattacharyya A, Chattopadhyay R, Mitra S, Crowe SE. Oxidative stress: an essential factor in the pathogenesis of gastrointestinal mucosal diseases. Physiol Rev 2014; 94:32954. doi: $10.1152 /$ physrev.00040.2012

[29] Kolgazi M, Cantali-Ozturk C, Deniz R, et al. Nesfatin-1 alleviates gastric damage via direct antioxidant mechanisms. J Surg Res 2015; 193:111-8. doi: 10.1016/j.jss.2014.06.057

[30] Chen SH, Liang YC, Chao JC, et al. Protective effects of Ginkgo biloba extract on the ethanol-induced gastric ulcer in rats. World J Gastroenterol 2005; 11:3746-50. doi: 10.3748/ wjg.v11.i24.3746

[31] EkinciAkdemir FN, Albayrak M, Çalik M, Bayir Y, Gülçin İ. The protective effects of $\mathrm{p}$-coumaric acid on acute liver and kidney damages induced by cisplatin. Biomedicines 2017;28;5 (2):18. doi: 10.3390/biomedicines5020018

[32] Lobo V, Patil A, Phatak A, Chandra N. Free radicals, antioxidants and functional foods: Impact on human health. Pharmacogn Rev 2010; 4:118-26. doi: 10.4103/09737847.70902 